\title{
La sélection des opérateurs privés par les services publics de l'emploi : comment sceller un accord?
}

The selection of private providers by public employment services: How might an agreement be concluded?

\section{Céline Remy}

\section{OpenEdition}

\section{Journals}

Édition électronique

URL : https://journals.openedition.org/ress/4513

DOI : $10.4000 /$ ress. 4513

ISSN : 1663-4446

Éditeur

Librairie Droz

Édition imprimée

Date de publication : 14 décembre 2018

Pagination : 183-208

ISSN : 0048-8046

Référence électronique

Céline Remy, "La sélection des opérateurs privés par les services publics de l'emploi : comment sceller un accord ? », Revue européenne des sciences sociales [En ligne], 56-2 | 2018, mis en ligne le 14 décembre 2021, consulté le 08 janvier 2022. URL : http://journals.openedition.org/ress/4513 ; DOI : https://doi.org/10.4000/ress.4513 


\title{
LA SÉLECTION DES OPÉRATEURS PRIVÉS PAR LES SERVICES PUBLICS DE L'EMPLOI: COMMENT SCELLER UN ACCORD?
}

\author{
CÉLINE REMY \\ Université de Liège \\ celineremy9@gmail.com
}

\begin{abstract}
Résumé. Suite aux directives européennes, les services publics de l'emploi belges délèguent une partie de leur mission d'accompagnement et de formation des chômeurs aux opérateurs privés d'emploi, à travers le dispositif d'appel à projets. La délégation de leur mission pose la question de la sélection des futurs prestataires. Cet article aide à comprendre comment les agents des services publics de l'emploi examinent les dossiers de candidature déposés par les opérateurs privés et, plus précisément, comment ils étayent leur décision à la suite d'opérations de jugement. L'analyse des données empiriques révèlera la manière dont les évaluateurs trouvent un accord lors du processus de sélection des opérateurs privés. D'un point de vue méthodologique, deux études de cas ont été effectuées au sein de services publics de l'emploi en Belgique francophone, à travers la réalisation d'observations participantes et d'entretiens semi-directifs auprès des agents des services publics et des prestataires.
\end{abstract}

Mots-clés: appel à projets, dossiers de candidature, jugement, prestataires de service, processus de sélection, services publics de l'emploi.

\begin{abstract}
Following the European directives, employment services delegate a part of their mission of support and training for the unemployed to private providers through calls for projects. The delegation of their mission raises the question of the selection of their future partners. This article helps to understand how agents of public employment services examine the applications submitted by private operators, and, specifically, how they support their decision based on acts of judgment. The analysis of empirical data reveals how these agents come to an agreement during the selection process for private operators. From a methodological point of view, two case studies were conducted in public employment services in the French-speaking part of Belgium. I used the methods of participant observation and semi-structured interviews carried out with agents of public services and private providers.
\end{abstract}

Keywords: applications employment services, call for projects, judgment, private providers, selection process. 


\section{INTRODUCTION}

Suite aux directives européennes', les services publics de l'emploi (SPE) doivent déléguer une partie de leur mission d'accompagnement et de formation des chômeurs (Divay, 2009) et partager ce marché avec les opérateurs privés (Rousseau, 2005). Ils deviennent des institutions régulatrices du marché de l'emploi (Georges, 2007). La délégation de leur mission engendre la question de la sélection des prestataires de service (Idem; Vivès, 20I3). C'est la manière dont cette sélection se réalise qui est l'objet de cet article, à partir d'une enquête conduite au sein de deux services publics de l'emploi opérant en Belgique francophone: Actiris en région bruxelloise et le Forem en région wallonne.

Auparavant et depuis les années 1990, ces deux SPE collaboraient uniquement avec les opérateurs non-marchands et associatifs, sur base d'une convention de partenariat gérée par le service juridique, renouvelée chaque année, avec un financement structurel à l'appui. Mais, la convention i8I de l'Organisation internationale du travail (OIT, 1997) sur les agences privées de placement a changé les pratiques en matière d'accompagnement des chômeurs. Elle invite désormais les SPE à collaborer avec les opérateurs marchands. Bien que ratifiée par la Belgique en 2004, cette convention amène les SPE à revoir leur méthode de recours au tiers et l'encadrement de ces prestataires au début des années 2000. Les SPE belges ont créé à cette période-là des services «partenariat» et des procédures pour formaliser et professionnaliser leurs pratiques.

Pour recourir aux opérateurs privés, la technique de l'appel à projets est mobilisée par le Forem depuis 2004 et par Actiris depuis 2008. Les opérateurs, qu'ils soient marchands ou non-marchands², sont mis en compétition régulièrement à travers des appels à projets biennaux ou triennaux qui leur permettent

I Le Livre blanc sur la croissance, la compétitivité et l'emploi (1993), la Stratégie européenne pour l'emploi et la Convention 18 I de l'Organisation internationale du travail sur les agences d'emploi privées (1997).

2 Les opérateurs privés non-marchands (ou associatifs) sont les associations sans but lucratif (ASBL), les organismes d'insertion socioprofessionnelle (OISP), les entreprises de formation par le travail (EFT), etc. tandis que les opérateurs marchands correspondent aux agences privées de placement, aux agences d'intérim, aux entreprises à responsabilité limitée (SPRL), aux sociétés anonymes, etc. 
de financer un ou de plusieurs projet(s) d'accompagnement et/ou de formation pour les demandeurs d'emploi. Actiris collabore avec en moyenne i Io prestataires et le Forem avec environ 210 prestataires, dont certains depuis des années. Les agents des services «partenariat» sont en charge de la sélection des projets déposés par les prestataires (rôle d'évaluateur) et du suivi ${ }^{3}$ de ces projets et leur évaluation (rôle d’accompagnateur).

Les agents des services «partenariat» ont pour mission de créer des partenariats et de veiller au respect des règles de collaboration. Lors de l'évaluation des dossiers de candidature, une tension fondamentale apparaît entre ces deux missions. Des dossiers présentent une ou plusieurs faiblesses, comme le fait de manquer d'une information, de posséder une incohérence ou une erreur, voire encore d'être de faible qualité. Ils ne respectent pas les critères donc ils devraient être rejetés, mais les agents souhaitent développer ou poursuivre la collaboration avec les opérateurs en question. Ou bien, un évaluateur donne un avis favorable au dossier et un autre défavorable.

Des débats naissent entre les agents à propos de la décision à prendre pour les dossiers et, surtout, pour la justifier auprès des opérateurs. Tout au long de la procédure de traitement des dossiers, les évaluateurs s'inscrivent dans un «enchevêtrement des registres argumentaires» (Cortéséro et al., 20I3). En focalisant l'attention analytique sur ces dossiers, nous poursuivons une intuition: le recueil et l'examen des données empiriques devraient révéler la manière dont les agents des SPE trouvent des accords - des arrangements et des compromis - pendant le processus de sélection des opérateurs privés avec lesquels ils vont nouer une collaboration.

D’un point de vue méthodologique, un travail d'observation a été effectué aux sein des SPE, dans les services «partenariat», et de comités de sélection. Suite aux observations effectuées, des entretiens semi-directifs ont été réalisés auprès des agents des SPE (les «évaluateurs », $\mathrm{N}=35$ ) pour cerner le vécu des

3 Pour d'amples détails sur l'activité d'encadrement des opérateurs privés par les agents des SPE dans le cadre des projets d'accompagnement et/ou de formation menés à destination des demandeurs d'emploi, voir Céline Remy (2013). 
négociations et des jugements octroyés aux dossiers de candidature. De plus, quelques prestataires $(\mathrm{N}=2 \mathrm{I})$ ont également été interrogés.

Larticle se structure comme suit: d'abord, le cadre théorique qui prend appui sur les concepts de règles, de «street-level bureaucrats», de compromis et d'arrangement est présenté. Puis, la méthodologie est exposée avec les SPE enquêtés et la méthode de collecte des données empiriques. Ensuite, le processus de sélection et des cas pratiques de dossier débattus en comité de sélection sont analysés en regard des éléments théoriques. Enfin, les résultats sont discutés avant de conclure.

\section{CADRE CONCEPTUEL ET THÉORIQUE}

Le processus de sélection des opérateurs privés par les agents des services partenariat des SPE contient plusieurs étapes avec de nombreux critères de sélection. Ces critères constituent des règles que les agents doivent appliquer lors de l'examen des dossiers de candidature. Cependant, ils font l'objet de multiples interprétations par les agents. En effet, si les règles orientent les conduites, elles ne les déterminent pas pour autant (Lascoumes, 1990). Non seulement le caractère général de celles-ci est borné par le particularisme de la situation, mais en outre sa signification n'est pas intégralement contenue dans l'énoncé (Batifoulier, 200I).

En ce sens, les règles sont envisagées comme des ressources pour l'action et non des contraintes. Selon Sidney Winter (1985, cité par Lascoumes 1990, p. 58), les règles ne seraient d'ailleurs pas faites pour être appliquées mais pour être négociées. Le postulat de base est que «la règle ne détermine pas la coordination, elle la facilite » (Favereau, 1998, p. 295). Or, si la règle ne constitue qu'un repère pour la coordination, elle suppose de la part des usagers une «attitude paradoxale de conformité critique » (ibid, p. 260). En ce sens, l'acteur est un être doté de réflexivité et d'une capacité interprétative. Il est capable d'interpréter son environnement (Chaserant et Thévenon, 200I) afin de requalifier les règles en fonction du contexte local d'application (Rébérioux et al., 200I, p. 254). 
S’intéresser aux débats entre agents des services «partenariat» des SPE lors de l'examen des dossiers de candidature pour saisir ces processus d'interprétation et de mise en application de la règle apparaît essentiel. Ces agents sont des «street-level bureaucrats» (Lipsky, 1980) en ce sens où ils ont un «pouvoir discrétionnaire» lors de la mise en œuvre de leur travail. Le pouvoir discrétionnaire désigne en droit le pouvoir reconnu de l'administration d'agir en se fondant sur sa propre appréciation, au-delà donc d'une simple application des règles mais en restant néanmoins dans un cadre légal (Dubois, 20I3). Le postulat d’un pouvoir discrétionnaire est d'autant plus difficile à limiter que l'encadrement hiérarchique n’a guère de prise sur un travail qui consiste en des interactions sur le terrain ou dans le huis clos d'un guichet (Lipsky, 1980).

En situation d'examen des dossiers, des débats naissent autour du jugement à porter aux dossiers et aux arguments de justification de la décision. Par moment, les agents des SPE doivent trouver un «compromis » ou un «arrangement» pour sortir de l'impasse, c'est-à-dire mettre un terme temporaire à l'échange interminable d'arguments. Ils font des «compromis» et des «arrangements », ce qui les amène à s'engager activement dans la définition du contenu concret des politiques publiques et d'avoir un «policy making role » (idem).

Le compromis consiste en le fait de se mettre d'accord pour composer, c'est-à-dire pour suspendre le différend, en renonçant à certains de leurs principes ou intérêts particuliers et en abandonnant la dispute (Nachi, 200I, p. I02). Le compromis est une «forme hybride d'accord» plus ou moins durable, au sens où il renvoie à plusieurs ordres de justification multiples dont sa visée est le bien commun ou la recherche d'un intérêt général (Nachi, 2007, p. 319). Les individus négociant le compromis font comme s'il existait un principe de rang supérieur capable de supporter une équivalence entre des objets relevant de natures différentes (Boltanski, 1990, p. 94).

Quant à l'arrangement, il ne fait pas référence à un «bien supérieur commun ». Il est une forme de relativisation qui n’a pas besoin des exigences de justice et échappe aux contraintes de justification (idem). En ce sens, Nachi stipule que dans de nombreuses situations courantes, les relations interpersonnelles entre membres d'un groupe s'appuient sur des arrangements plutôt 
que sur les compromis. La concession qui est faite dans l'arrangement consiste précisément à ne pas remonter jusqu'à un principe de justice (Boltanski et Thévenot, 1991, p. 163). Les individus s'arrangent entre eux sans mettre un terme définitif au conflit. Le différend n’est que temporairement apaisé.

\section{MÉTHODOLOGIE DE RECHERCHE}

\section{I. DEUX SERVICES PUBLICS DE L'EMPLOI ENQUÊTÉS}

Deux services publics de l'emploi ont été étudiés en Belgique francophone à savoir Actiris à Bruxelles et le Forem en Wallonie. Le dispositif d'appel à projets (voir Figures I et 2 en Annexe) leur permet de planifier l'affectation de plus ou moins 5000 places-stagiaires pour les 120000 chômeurs bruxellois et de réserver environ 8000 places-stagiaires pour les 200000 chômeurs indemnisés en région wallonne. Concrètement, Actiris organise un appel à projets par mesure d'accompagnement (mobilisation et orientation, mesure courte d'orientation, recherche active d'emploi, création d’activité, etc.), alors que le Forem propose un large appel pour l'ensemble de ces mesures. Les projets déposés par les prestataires peuvent concerner des profils particuliers de chômeurs (analphabètes, jeunes, seniors, femmes, etc.).

Un cahier des charges, diffusé sur le site internet des SPE, précise les modalités de soumission et les critères d’appréciation des dossiers de candidature. Les agents du service «partenariat», dénommés «gestionnaires de projets» chez Actiris (30 sur 940 employés au moment de l'enquête) et «chargés de relations partenariales » au Forem (I40 sur 4000 employés répartis entre le siège central et les i directions régionales au moment de l'enquête), ont pour mission de sélectionner les dossiers de candidature (dénommés «évaluateurs» dans ce rôle-là) et par la suite d'encadrer les opérateurs privés dans la mise en ouvre de ces projets à destination des demandeurs d'emploi. Le Forem reçoit pour l'appel qu'il organise sur trois ans environ huit cents dossiers dont un peu plus de la moitié est financée. En revanche, Actiris réceptionne au maximum trente dossiers par appel à projets dont les trois quarts sont généralement subsidiés. 


\subsection{LA COLLECTE DE DONNÉES EMPIRIQUES}

Les services «partenariat» ont établi des procédures et des critères pour effectuer l'évaluation des dossiers de candidature qui sont précisés dans une série de documents : le canevas et le guide administratif et financier de l’appel à projets, le modèle type du dossier de candidature, la fiche d'éligibilité et les grilles d'analyse des dossiers. Nous nous sommes procurés ces documents pour en prendre connaissance ainsi que ceux liés aux précédents appels à projets pour en cerner les changements et les évolutions.

Un travail d'observation a d'abord eu lieu aux différentes étapes de l'appel à projets. De la sorte, nous avons assisté aux réunions de préparation (séance d'information aux opérateurs par les agents des services partenariat, séance entre évaluateurs pour la planification du travail d'évaluation des dossiers et rappel des spécificités de l'appel à projets) et à certains comités de sélection organisés entre évaluateurs pour discuter de l'éligibilité et de la cohérence/pertinence des dossiers. Il ne nous a pas été autorisé à participer aux comités de gestion des SPE qui président à la dernière étape de validation des dossiers.

Ce travail d'observation a été réalisé entre mai 20II et avril 20I2. Il a permis d'identifier les difficultés qu'il y a à poser un jugement et à en justifier la décision. À partir des éléments collectés lors de l’observation, des entretiens semi-directifs ont été réalisés auprès des évaluateurs au sein du service partenariat d'Actiris $(\mathrm{N}=\mathrm{I} 3)$ et du Forem $(\mathrm{N}=22)$ pour cerner le vécu des négociations et des jugements octroyés à ces dossiers. Nous avons également interrogé quelques prestataires du Forem $(\mathrm{N}=\mathrm{I}$ ) $)$ et d’Actiris $(\mathrm{N}=\mathrm{I}$ ) sur leur expérience d'introduction de projets auprès du SPE et le déroulement de la collaboration avec lui. Tant les éléments d’observation que les entretiens seront utilisés dans la partie empirique. 


\section{DESCRIPTION ET ANALYSE DES DONNÉES EMPIRIQUES}

\section{I. LA PROCÉDURE DE SÉLECTION}

Le processus d'examen des dossiers de candidature comporte deux étapes : la phase d'éligibilité et la phase d'évaluation du contenu du projet (cohérence et pertinence). Suite au travail de lecture, entre huit et quinze évaluateurs se réunissent en comité de sélection (un par mesure d'accompagnement ou de formation) pour passer en revue les avis octroyés aux dossiers. Au Forem, les comités d'éligibilité et de cohérence des dossiers sont dirigés par le siège central en présence d'un représentant de chaque direction régionale. L'étape d'analyse de la pertinence du dossier s'effectue en interne des directions régionales. Du côté d'Actiris, le service «partenariat» dirige les comités de sélection (éligibilité et cohérence/pertinence) et invite d'autres services du département «chercheur d'emploi » du SPE à savoir le service «qualité » et le service «guidance» à les aider dans cette tâche d'analyse des dossiers.

Au moment de l'éligibilité, les évaluateurs vérifient la validité ainsi que la complétude du dossier et des pièces administratives et juridiques. Le dossier est éligible s'il a été introduit par envoi recommandé et sous format électronique à date prévue et ce, avec le bon modèle de candidature, s'il est entièrement complété et correspond à la mesure d'accompagnement définie par l'appel à projets, s'il contient une identification claire du porteur de projet et du territoire d'action et s'il est à $80 \%$ à destination des chômeurs visés par l'appel et gratuit pour ceux-ci. Les pièces administratives demandées sont: les statuts, le bilan financier sur les trois dernières années, les documents de l'Office national de sécurité sociale, de précompte professionnel et de la TVA (le cas échéant), le document attestant une comptabilité analytique ou séparée sous forme informatisée, un organigramme, le curriculum vitae des personnes affectées au projet et un document de non faillite/liquidation de l'organisme.

Les évaluateurs se réunissent en comité de sélection pour discuter des dossiers. Parfois, presque l'ensemble des dossiers est critique, notamment en raison de documents manquants. Les débats entre agents doivent déboucher sur l'éligibilité ou l'inéligibilité du dossier. Dans le premier cas, le dossier passe 
à la phase d'évaluation du contenu qui consiste en un examen de la cohérence et de la pertinence du projet. Dans le second cas, le service «partenariat» (du siège central pour le Forem) envoie la décision par courrier à l'opérateur qui a toujours l'opportunité de déposer un recours s’il estime la décision injuste.

Ensuite, les agents évaluent l'adéquation du projet d’accompagnement et/ou de formation des chômeurs aux besoins socio-économiques et aux priorités ${ }^{4}$ de la région, le degré d’adéquation du projet aux mesures d’accompagnement/formation telles que définies dans le cahier des charges (adéquation des objectifs poursuivis, du public visé, des résultats attendus), la qualité et le réalisme du projet ainsi que la disponibilité et la qualité des ressources (humaines, matérielles, techniques, etc.) du prestataire pour atteindre les objectifs (mesurés par la technique d'évaluation des participants et du projet; la fourniture d'un délivrable pour chaque candidat qui peut-être une attestation d'emploi ou de formation qualifiante, un bilan de compétences, un projet professionnel, etc.), l'expérience du prestataire dans l'accompagnement des demandeurs d'emploi (déroulement des prestations antérieures s'il y en a eues) et le prix demandés.

Le dossier est lu par au minimum deux évaluateurs qui attribuent chacun une appréciation. Il est avalisé très rapidement sans discussion quand il possède deux avis positifs ou deux avis négatifs. En revanche, les dossiers contenant des avis opposés ou ne respectant pas certains critères font l'objet de débats. Parfois, plus de la moitié des dossiers sont concernés. Les discussions peuvent avoir pour conséquence d'interroger les «qualités» attribuées aux dossiers précédents. De nouvelles discussions s'ouvrent sur la qualification de ces derniers quand les évaluateurs estiment avoir été injustes. L’ensemble des décisions, au travers d’un classement des dossiers, sont envoyées au comité de gestion (composé de l'administrateur délégué du SPE, de responsables du service partenariat, des représen-

4 Les priorités sont définies à partir des besoins de la région en termes, par exemple, de secteur d'activités, de répartition géographique, de public-cible, de priorisation des mesures de l'appel à projets, de priorités aux nouveaux opérateurs, etc.

5 Le prix est balisé par le service partenariat. L'opérateur peut être rémunéré par individu accompagné (forfait d'un minimum de 650 euros selon la durée de la mesure d'accompagnement ou de formation), soit par heure d'accompagnement ou de formation donnée (montant d'environ 12 euros). 
tants de la région et, parfois, d'experts externes universitaires) qui valide ou invalide le jugement des dossiers de candidature. Le comité de gestion peut demander des éclaircissements lorsque l'argumentation n’est pas suffisamment claire.

Le service «partenariat» a pour mission de rédiger et d'envoyer la lettre de réponse aux opérateurs. Les évaluateurs du Forem récoltent un maximum d'arguments pour justifier la décision prise tandis que leurs homologues chez Actiris utilisent un système de cotation. Ils sont parfois amenés à poser des questions d'éclaircissement à l'opérateur en cours de procédure. Les évaluateurs peuvent aussi suggérer ou imposer des conditions à l'opérateur pour que son dossier de candidature soit accepté, telles que la réduction ou l'augmentation du nombre de demandeurs d'emploi à encadrer, la suppression ou l'ajout de certains modules dans le projet, la réduction du budget, ou autre.

\subsection{LA RÉCEPTION DU DOSSIER: DES PRATIQUES DIVERGENTES}

Un des critères d'éligibilité concerne l'envoi du dossier de candidature sous pli recommandé à une date butoir. Certains opérateurs ont envoyé leur dossier sous pli normal ou l'ont déposé en main propre au sein du SPE. Les évaluateurs du côté d’Actiris décident de rejeter l'unique dossier envoyé sous pli normal, parmi les quatorze dossiers réceptionnés. La règle est appliquée de façon drastique sans mériter l'ouverture d'un débat.

Par contre, la prise de décision se déroule différemment du côté du Forem. Une trentaine de dossiers sur les huit cents reçus soulève des difficultés car certains sont envoyés sous pli normal, après le délai fixé ou déposés en main propre avec un accusé de réception donné par l'agent. Cela suscite de vives discussions entre les évaluateurs car ils réalisent la présence de pratiques différentes entre les directions régionales, qui entrainent le non-respect des prescrits de l'appel à projets.

[Siège central] : le recommandé est un critère qui prémunit l'Office. Si l'opérateur a envoyé son dossier par courrier normal et qu'il dit avoir envoyé dans les délais, tant pis pour lui. Il faut que l'Office puisse prouver que le dossier est arrivé dans les temps (avec timbre et accusé de réception).

[Direction régionale 3] : ça ne va pas si on accepte certaines choses dans une direction régionale et pas dans une autre. 
[Direction régionale 5] : ça pose vraiment question.

[Siège central]: on a déjà eu des plaintes d'opérateurs qui disaient qu'on avait accepté un dossier alors qu'il n'était pas envoyé par recommandé. Ils auraient pu alors eux aussi éviter de payer des recommandés.

[Direction régionale 4] : alors, on décide de classer ces dossiers non éligibles.

[Direction régionale 5]: on a refusé dans le dernier appel l'opérateur Y car il n’avait pas envoyé par recommandé alors que c'est un bon opérateur.

[Siège central] : tout le monde est d'accord avec le fait de rendre les dossiers non éligibles?»

Au bout d'un bon moment de discussion, le siège central et plusieurs directions régionales (la 3, 4 et 5) semblent s'accorder sur une décision commune de rendre non éligible les dossiers de candidature non envoyés sous pli recommandé. L’argument du respect de la règle est mis en avant, permettant ainsi d'honorer la valeur de l'équité par rapport aux autres opérateurs qui ont introduit leur dossier sous le format demandé. Cette solution est en inadéquation avec la mission de «réseautage » du SPE, car elle exclut un ensemble d'opérateurs du conventionnement avec le SPE. Elle agrée les agents qui ont appliqué la règle à la lettre mais elle met en mauvaise posture ceux qui devront annoncer aux opérateurs l'inéligibilité de leur dossier alors qu'ils leur ont fourni un accusé de réception.

[Direction régionale 7]: on veut bien accepter cette décision pour les envois courrier normaux mais pas pour les dossiers remis en main pour lesquels on a donné un accusé de réception. On n’a pas fait attention à ça car les opérateurs sont venus nous trouver. On ne va pas reprocher aux opérateurs de se déplacer pour nous apporter leurs dossiers.

[Direction régionale 5] : si on supprime le recommandé, on va devoir au prochain appel passer notre temps à remplir des accusés de réception.

[Siège central] : si on accepte un dossier par accusé de réception, il n'y a pas de différence avec le courrier normal d'un point de vue juridique. On peut encore jouer sur des questions techniques : signaler à l'opérateur qu'il n’a pas respecté le prescrit et que la décision sera prise par le comité de gestion.

[Direction régionale 5]: en plateforme partenariale avec les opérateurs, on a bien stipulé que les dossiers non recommandés ne seraient pas lus. 
[Direction régionale 3] : il faut alors rendre le dossier inéligible plus tard.

[Siège central] : on peut faire intervenir le comité de gestion alors on envoie un courrier à l'opérateur où on stipule que le comité décidera d'accepter ou de refuser le dossier. On peut mettre dans la convention que l'opérateur doit absolument respecter le prescrit la fois prochaine.

[Direction régionale 5] : certains opérateurs sont champions pour ne pas respecter les prescrits. Ça ne fonctionnera jamais cette clause.

[Direction régionale 3] : c'est une chance quelque part que ces opérateurs n’aient pas respecté le prescrit car ce sont des dossiers foireux donc c'est l'occasion de s'en débarrasser.

[Siège central] : on ne veut pas mettre à mal certaines directions régionales.

[Direction régionale 7]: on est dans une mauvaise posture car on ne va pas aller dire aux opérateurs que leur dossier est refusé parce qu'on l'a accepté avec accusé de réception. On les connaît très bien ces opérateurs, depuis longtemps parfois.

[Direction régionale 5] : logiquement, vous auriez dû leur dire d'envoyer par recommandé. C'est un document officiel.

Le débat laisse transparaître la posture plus ou moins drastique de chaque direction régionale en matière d’application des règles. Les dossiers déposés en main propre au sein de la direction régionale posent le plus question aux évaluateurs. Les agents de la direction concernée mettent en exergue les collaborations rapprochées qu'ils ont avec les opérateurs (la proximité, la familiarité, les bonnes relations, la connaissance interpersonnelle de longue date parfois) ainsi que le côté spontané dans leur pratique.

Par ailleurs, une autre direction régionale souligne le souhait de rendre inéligible les dossiers envoyés par courrier normal car ces opérateurs-là sont peu fiables et ont mauvaise réputation (récurrence au niveau des difficultés relationnelles, de gestion, d'encadrement des candidats, etc). Une solution proposée par le siège central est de remettre la décision dans les mains du comité de gestion et de rédiger une clause spéciale dans la convention de partenariat. Mais, tous les évaluateurs ne sont pas satisfaits de cette suggestion. De plus, ils mentionnent que le durcissement des règles n’a pas d'effet sur le comportement de certains opérateurs. 
[Siège central] : on ne peut pas créer une inégalité.

[Direction régionale 5]: on s'arrête où si on accepte cela?

[Direction régionale 6]: je trouve ça grave. Si on était dans le privé, ces dossiers seraient refusés.

[Siège central]: on a les arguments pour refuser ces dossiers mais ça peut poser problème pour certaines directions régionales au niveau de la crédibilité et de leur image. Si on accepte les dossiers avec accusé de réception et pas les dossiers sous pli normal, comment peut-on prouver juridiquement cette différence?

[Direction régionale 5]: on peut retrouver les dossiers envoyés sous pli recommandé ou déposé avec accusé de réception car ils sont répertoriés dans la base de données, ce qui n'est pas le cas des autres.

[Direction régionale 3]: on précise aux opérateurs qu'ils doivent vérifier la grille d'éligibilité avant d'envoyer leur dossier. Ils ne le font pas puis on se retrouve à discuter de leur acceptation.

[Direction régionale 8]: on doit reconnaitre que l'on a fait une erreur.

[Direction régionale 4]: on doit reconnaitre que l'Office a fait une erreur et donc accepter tous les dossiers.

[Direction régionale II ] : on aimerait bien qu’il soit inéligible ce dossier-là (rire).

[Siège central]: on ne peut pas faire ça. Juridiquement, ça nous pose moins de problème d'accepter les dossiers que de les refuser. Il faut que l'on règle ce problème pour l'année prochaine, que l'on mette une note en rouge sur le document.

[Direction régionale 5]: on vous [au siège central] envoie les autres opérateurs s'ils râlent parce que leur dossier n'est pas accepté.

[Siège central]: pour les deux cas (courrier normal et accusé de réception à la main), on les accepte.

Pris entre les questions d'équité entre opérateurs, de respect des règles du cahier des charges de l'appel à projets et de crédibilité (d'image) des directions régionales dans leur démarche (fournir un accusé de réception pour un dépôt en main propre) vis-à-vis des opérateurs, le siège central suggère de choisir une solution plus tenable juridiquement qui est d'accepter les dossiers arrivés dans les temps et ce, qu'elle que soit la méthode d’envoi. En conséquence, tous les dossiers concernés sont considérés comme éligibles. L'esprit 
de la règle est respecté mais la lettre - l'envoi recommandé - est négociée pour s'étendre à d'autres modalités de dépôt.

Les agents qui ont commis une erreur en acceptant les dossiers en main propre sont soulagés de ce compromis car leur direction régionale ne sera pas décrédibilisée face aux opérateurs. Puis, ils pourront potentiellement collaborer avec ces derniers si les dossiers répondent aux autres critères de sélection. En revanche, les évaluateurs qui souhaitaient l'application drastique de la règle s'interrogent sur l'utilité d'instaurer préalablement des critères s'ils ne sont pas respectés et sur les limites de leur assouplissement. Pourtant, ce compromis apparaît le plus adéquat car il permet de dissimuler l'erreur des agents, d'être «juste» par rapport aux prestataires qui ont été «autorisés » à déposer en main propres et d'éviter les plaintes, en tout cas de ceux-là.

\subsection{LE PROGRAMME D'ACCOMPAGNEMENT OU DE FORMATION Un problème d'heure}

Dans le dossier de candidature, les opérateurs privés doivent fournir une méthode d'encadrement des demandeurs d'emploi ainsi qu'un programme d'activité ou de formation. Le cahier des charges de l'appel à projets contient des critères à respecter pour introduire un dossier dans telle ou telle mesure d'accompagnement ou de formation. En cas de problème d'heure (trop ou trop peu) dans le programme, certaines heures peuvent être négociées alors que d'autres non, comme le montrent les exemples ci-dessous.

[Siège central] : à priori, le dossier ne serait pas à retenir.

[Direction régionale 8]: il faudrait mettre une condition en termes de garantie de moyen. Le programme est très succinct et le stagiaire doit choisir entre différents modules.

[Siège central] : l'Office n'a pas le droit de donner une condition sur la méthodologie. C'est de la responsabilité de l'opérateur. L'office doit regarder les moyens pour garantir l'atteinte des objectifs.

[Direction régionale 8]: c'est l'effet pervers de l'optionnel. Le nombre d'heure ne tient pas la route. Ça pose question sur le suivi des stagiaires. 
[Siège central]: il ne faut pas confondre les arguments. On ne peut pas justifier sur le volume d'heures.

[Direction régionale 8]: c'est un tout nouveau dossier, on ne va pas le refuser.

[Siège central] : le premier argument, c'est qu'il n'y a pas assez d'heures de stage (c'est seulement une journée d'observation en entreprise) et le deuxième, c'est que le module permis de conduire est trop court. On ne peut pas se prononcer dessus car l’optionnel est autorisé.

[Direction régionale 8] : l'argument est faible pour justifier un refus.

[Siège central]: on n'est pas là pour réagir affectivement mais voir si le cadre légal est appliqué. La Direction régionale 8 veut-elle retenir le dossier?

[Direction régionale 8]: oui, en mettant comme recommandation de mettre un stage d'observation pour tous et de préciser le contenu de tous les modules.

Au premier abord, le dossier de candidature n'est pas à retenir car il contient un problème d'heures. Mais, les agents trouvent un arrangement entre eux par la mise en exergue d'arguments en faveur du dossier, notamment son caractère innovant. L'opérateur est nouveau, donc il n'est pas familier avec toutes les exigences d'une collaboration avec le service public de l'emploi. Une chance lui est laissée de mener son projet, en respectant deux recommandations.

La recherche d'un arrangement peut échouer quand les agents n'arrivent pas à mener à bien leur négociation, comme c'est le cas ci-dessous pour un autre dossier examiné par les évaluateurs du Forem.

[Direction régionale 5] : le projet est cohérent car il est à l'identique mais l'opérateur s'est trompé. Il a diminué son projet de quatre heures donc il se déroule sur vingt-quatre heures.

[Siège central] : il est de vingt-quatre heures donc il est inéligible car la mesure d'accompagnement est de minimum vingt-cinq heures.

[Direction régionale 5]: c'est un opérateur qui ne roule pas. Il a diminué ces heures pour bien coller à la réalité. Il fait bien son travail avec les demandeurs d'emploi. Et, on va le refuser car il manque une heure? Il a respecté tous les autres critères donc s'il avait gardé ces vingt-huit heures initialement prévues mais qu’il dépassait le délai des trois mois, comme pour les autres dossiers, le dossier serait passé alors?

[Siège central] : certainement. 
L'opérateur a introduit un dossier où l'action de formation des demandeurs d'emploi dure vingt-quatre heures. Seulement, le cahier des charges de l'appel à projets impose une durée de vingt-cinq heures. Le dossier est déclaré inéligible. Un des évaluateurs manifeste son désaccord par rapport à cette décision car de précédents dossiers, introduits dans d’autres mesures de formation, ont été acceptés avec une durée de prestation erronée, trop longue cette fois-ci de trois jours. Pourtant, le cahier des charges mentionne un étalement de la mesure de formation sur trois mois maximum. Les évaluateurs ont révisé la règle dans le cas des trois mois, en y ajoutant les trois jours supplémentaires, mais pas pour celui des vingt-cinq heures.

L'agent souhaitant un changement de la décision est déçu car il voulait absolument obtenir une décision positive pour le dossier des vingt-quatre heures. La règle est appliquée de façon drastique. Le dossier est refusé pour l'heure manquante. L'évaluateur n'arrive pas à faire valoir ses arguments qui s'appuient principalement sur la connaissance interpersonnelle de l'opérateur et sur son travail de qualité avec les demandeurs d'emploi. Pour avoir un dossier conforme aux règles de l'appel à projets, l'opérateur doit ajouter une heure dans son programme, ce qui change le contenu de la mesure de formation.

Les agents ne peuvent pas modifier l'esprit de la mesure. Accepter le dossier en l'état dénature, hélas, le contenu de la mesure. Dans le cadre de la durée d'étalement des trois mois, les évaluateurs ne touchent pas au programme initial. Ils ne font que repréciser les modalités du cadre dans lequel la session de formation doit se dérouler. De la sorte, c'est uniquement la lettre qui est quelque peu modifiée et non l'esprit de la mesure. Aucun arrangement ni compromis n'est fait.

\subsection{LA QUALITÉ SOUS TENSION: CONTENU DU DOSSIER VERSUS PRESTATIONS ANTÉRIEURES}

Dans l'analyse des dossiers, les agents regardent la cohérence et la pertinence du projet proposé par l'opérateur. Ils évaluent la qualité du dossier en regard de son contenu mais aussi de la qualité des prestations antérieures, s'il y en a eues. Une tension peut naître entre ces deux critères. 
Un exemple emblématique, du côté du Forem, est celui d'un prestataire de longue date qui a introduit un dossier bien rédigé, selon les évaluateurs, mais qui n’a pas rempli ses obligations en termes de gestion administrative des demandeurs d'emploi lors de la précédente collaboration. Par la signature d'une convention de partenariat, l'opérateur doit lui envoyer des documents administratifs en vue de la rétribution (un euro de l'heure) des stagiaires pour leur participation à la formation. Le manquement à cette obligation a provoqué des retards de paiement et, par conséquent, des plaintes de la part des demandeurs d'emploi touchés par cette situation.

[Direction régionale 5]: on ne peut pas revoir le programme? Ça fait des années qu'on travaille avec lui et, malgré tout ce qu'on a mis en place, on a constaté une dégradation dans la collaboration et le suivi des projets. On s'est retrouvé avec des plaintes de stagiaires.

[Siège central] : il y a de l'orientation et un bilan de compétences donc c'est bon. On regarde la cohérence du dossier, pas la pertinence qui est l'étape d'après. Vous pourrez vous arranger pour mettre le dossier en fin de liste, qu'il ne soit pas financé.

[Direction régionale 5]: j'essaie d'être objective pour ce dossier mais, je suis trop imprégnée du vécu. Personne ne sait se positionner par rapport à cet opérateur. Il a fait des jolies phrases où on ne peut rien lui reprocher dans le dossier. Il est malin comme l'opérateur M. Le dossier est entre la mesure «formation générale » et «emploi salarié».

[Siège central] : il sera alors à retenir sous condition? Avoir un délivrable pour chaque candidat encadré.

[Direction régionale 5] : l'opérateur a oublié de cocher la case bilan de compétences. On n'a pas de délivrables sur lequel sappuyer pour requalifier le dossier dans l'une ou l'autre mesure.

[Siège central] : il est à retenir sous condition. Il faut des délivrables intégrant le bilan de compétence et un programme très axé sur la formation générale plutôt que la mobilisation.

Lors d'une rencontre ultérieurement avec l'agent, il précise que le dossier a finalement été rejeté ultérieurement en phase de pertinence : 
On l'a refusé en phase de pertinence car ça fait des années qu'on travaille avec lui et, malgré tout ce qu'on a mis en place, on a constaté une dégradation dans la collaboration et le suivi des projets. On s'est retrouvé avec des plaintes de stagiaires. Avec de tels éléments, on argumente contre le dossier même s'il est bien écrit. Ces éléments objectifs permettent d'éviter le délit de faciès. Je ne peux pas dire «il est insupportable ». On s'était dit qu'on allait demander aux opérateurs qui sont sur tout le territoire de la région wallonne de se centrer sur quelques sous-régions, de ne pas être partout, pour apprendre à mieux connaitre le tissu local et mieux s'implanter. J’avais demandé que l'opérateur Y ne soit pas sur mon territoire parce que, à un moment, c'est devenu épidermique tellement c'était pénible. Mais, en effet, on ne peut pas dire uniquement sur base de ça qu'on les refuse. (Agent M. du Forem 5).

Tout au long du suivi du projet, l'agent a reprécisé les règles du conventionnement à l'opérateur en question. Il a également proposé des pistes de solution mais rien n’a changé. Le prestataire n’a pas manifesté de bonne volonté dans les changements que l'agent attendait de lui. Sur la base des évaluations antérieures mettant en avant ces faits, les évaluateurs de la direction régionale 5 décident, en phase de pertinence, de ne pas retenir le dossier. Ils s'arrangent pour ne pas rendre ce projet important sur leur territoire et mettre en avant les plaintes des candidats.

Du côté d'Actiris, des évaluateurs doivent examiner des dossiers de candidature dans le cadre d'un appel à projets conjoint avec un autre service public, Bruxelles Formation. La procédure veut que chaque institution évalue la partie du dossier la concernant: le volet formation pour Bruxelles Formation et le volet accompagnement des demandeurs d'emploi pour Actiris. Les deux institutions doivent fournir un avis positif pour que le dossier soit accepté. Après lecture, deux dossiers ont obtenu un avis négatif de la part d'Actiris et un avis positif de la part de l'autre institution. Selon la règle établie, le dossier doit être rejeté. Cependant, lors du comité de sélection, les évaluateurs ont décidé de lire le dossier rendu par l'opérateur à l'autre institution pour comprendre les avis de chacun. La règle de départ est aménagée en cours de traitement des dossiers car l'un d'entre eux pose question aux évaluateurs. 
On sait que c'est un opérateur qui travaille bien mais on ne le voit pas dans son dossier de candidature. Il n’a pas été très explicite. Mais il faut partir du principe que le comité de sélection n’est pas supposé connaître l'opérateur. Mais évidemment, on le connaît. Il y a une rubrique «expérience avec l'opérateur» mais on ne donne pas de cote pour ça. Les résultats des trois années précédentes sont positifs mais on ne le voit pas dans le dossier. C'est la deuxième fois qu'il fait ça. Le comité de sélection a voulu lui donner le signal comme quoi ça n'allait pas. On a dit «négatif ». On a fait un deuxième comité de sélection pour le laisser passer en mettant une condition qui est de faire une évaluation après un an. Il a une convention pour un an alors que les autres ont une convention pour trois ans. Si ça ne va pas, il ne sera pas reconduit. Toutes les subventions vont tomber. (Agent C. d'Actiris)

Les évaluateurs ont décidé de donner une deuxième chance à l'opérateur tout en lui imposant un cadre de collaboration plus strict. Ils l'ont accepté car ce dernier a des résultats positifs depuis trois ans. Ainsi, la présence d'une évaluation positive lors de la précédente collaboration est un élément qui joue en faveur de l'acceptation du dossier de l'opérateur. Les résultats sont évoqués par les examinateurs sous deux aspects: le quantitatif et le qualitatif qui alimentent positivement ou négativement la réputation de l'opérateur; dans ce cas-ci positivement.

C'est la deuxième fois que l'opérateur introduit un dossier qui ne reflète pas le travail fourni antérieurement. L'évaluateur lui avait donné des conseils mais il n'en a pas tenu compte pour la rédaction du dossier. Malgré cela, le dossier est accepté sur la base des résultats obtenus lors des anciennes collaborations. Ces dernières offrent des garanties au niveau des résultats. Le fait d'être un ancien partenaire, d'être connu du service «partenariat», et d'avoir une bonne réputation, entre autres, au niveau de la qualité de l'accompagnement des demandeurs d'emploi sauve le dossier.

Un opérateur inconnu, dans une situation similaire, aurait certainement eu moins de chance d'être repris car aucune évaluation antérieure n’aurait pu appuyer le dossier en sa faveur. La renommée est plutôt fondée, ici, sur la qualité du travail que sur la relation entre le SPE et le partenaire car ce dernier n’est pas toujours très collaborant. Plusieurs évaluateurs ont précisé la difficulté de gérer la relation au quotidien avec le partenaire en matière de gestion admi- 
nistrative et financière et, le souhait de lui faire comprendre l'importance de saméliorer dans la rédaction de ses futurs dossiers de candidature.

Finalement, il est partenaire mais il est furieux car son dossier n'a pas de bons points chez nous. Au téléphone, un agent du service lui a donné des explications parce qu'apparemment, c'est à chaque fois la même chose avec ce partenaire-là. On va faire une évaluation après un an. À chaque fois, il introduit des dossiers, de beaux dossiers mais on veut qu'il comprenne qu'il doit améliorer la rédaction de ceux-ci [...]. Les contacts avec lui sont mauvais. Et, c'est très difficile de travailler avec lui pour l'encodage des candidats et les dossiers financiers. Apparemment, d'autres services d'Actiris ont beaucoup de problèmes avec lui mais ça n'a pas influencé leur décision par rapport au dossier. (Agent D. d'Actiris)

Malgré le manque de professionnalisme dans la rédaction du dossier de candidature, les évaluateurs l'acceptent tout de même en faisant un compromis. La présence de bons résultats obtenus par le passé prédomine par rapport au manque de rigueur mais aussi de sympathie dans les relations quotidiennes avec les agents. Le dossier de candidature est un peu faible et l'opérateur n'a pas tenu compte des conseils donnés antérieurement par les agents, ceux-ci décident de le recadrer, en imposant notamment une évaluation intermédiaire pour définir la poursuite ou non du partenariat. Les évaluateurs font un compromis où ils imposent de nouvelles règles à l'opérateur, comme une évaluation après un an de collaboration.

\subsection{LE PRIX DU PROJET: LE CRITÈRE FINAL}

En fin de processus de sélection, les évaluateurs analysent le prix demandé par l'opérateur pour la réalisation de la prestation (frais de locaux, de poste(s) de formateur, de matériel et/ou matériaux). Ils centrent d’abord leur attention sur le contenu du dossier de candidature avant d'en examiner le prix. Un certain budget est attribué pour chaque mesure en fonction du nombre de places à ouvrir pour les chômeurs. Le prix n'est pas négocié en tête à tête entre les agents du SPE et l'opérateur. Ce dernier fait une proposition de budget que le SPE accepte ou modifie si le prix est trop élevé. 
Certains prestataires demandent un budget exorbitant ou trop faible. Dans le cas du budget surestimé, les évaluateurs s'interrogent sur un éventuel acte opportuniste de la part de l'opérateur car ils ne comprennent pas les raisons d'un tel budget. Les prix exorbitants sont recadrés grâce aux systèmes de balises mis en place par les SPE (calcul de la médiane de tous les prix des projets). Dans le cas du budget sous-estimé, les évaluateurs craignent la non viabilité financière du projet mais aussi de l'organisme d'insertion sur le long terme. Certains opérateurs n'évaluent pas correctement la valeur financière de leur prestation. Dans les deux cas, les évaluateurs se posent alors la question de la fiabilité de ceux-ci.

Quelques extraits de commentaire d'évaluateurs d'Actiris ci-dessousà propos de trois dossiers à juger :

\section{Dossier 1.}

[Service "partenariat» I] : il demande une dérogation qu'il a déjà eue auparavant donc on devra lui autoriser. On ne peut pas revenir en arrière. Ça se passe très bien donc on ne peut pas leur retirer. Il ne faut pas oublier que c'est l'opérateur Z!

[Service «qualité»] : je ne suis pas d'accord avec toi. Je trouve que le dossier est peu clair. La méthodologie est peu approfondie surtout au niveau des séances. Il y a toute une partie de la méthodologie qui n’est pas appropriée. Il faut supprimer certaines phases.

[Service «partenariat» 2] : la temporalité me semble bonne donc le fait de voir les personnes à intervalle régulier. Il faut voir si ça convient pour ce type de public.

[Service «partenariat» I] : au niveau du budget, ça ne va pas. Il y a un cumule.

\section{Dossier 2.}

[Service «partenariat» I] : le dossier n'est ni bon ni mauvais. Il est long dans la durée. Il y a deux groupes de quatre semaines. Le budget me semble faible. Il faut vérifier car ce n'est pas clair donc il faut envoyer un mail à l'opérateur.

\section{Dossier 3.}

[Service «partenariat» I] : il a déjà engagé les personnes. Ce partenaire a perdu un subside par ailleurs. Mais, Actiris ne doit pas être un complément d'un subside perdu. 
Du côté du Forem, les dossiers sont d’abord classés selon les priorités de la direction régionale et, ensuite, selon le nombre de commentaires qui leur sont attribués. La direction régionale financera les projets tant qu’elle a du budget. Ainsi, certains dossiers en fin de liste risquent de ne pas être financés. Le critère du prix intervient en tout fin du processus de sélection.

Le dossier est passé à la trappe parce que c'était trop exagéré au niveau du budget qu'il demandait. Je vais rencontrer l'opérateur pour lui expliquer. Il ne comprenait pas pourquoi il n'était pas repris. Ça fonctionne dans d'autres directions régionales car elles préfèrent un opérateur qui prend les gens par la main mais nous, on préfère autre chose donc on ne l’a pas financé. (Agent J. du Forem 6).

Dans les arguments des évaluateurs, la question du prix vient s'articuler à d’autres aspects du dossier, comme la qualité du projet ou de l'encadrement des candidats, la qualité de la rédaction, le déroulement des prestations antérieures mais aussi aux priorités de la région (ou direction régionale). Tous ces aspects alimentent «l'étiquette réputationnelle» de l'opérateur de manière positive ou négative, élaborée par les évaluateurs et qui leur permet par conséquent d’accorder ou non leur confiance. La bonne évaluation du prix joue en faveur de sa réputation, ce qui n'est pas le cas quand le prestataire est dans la démesure concernant l’aspect financier du projet. Elle constitue un élément supplémentaire qui permet aux évaluateurs d'avoir confiance en l'opérateur et d'établir un partenariat avec lui. À partir de tous ces éléments, les évaluateurs sarrangent pour ordonner les projets qu'ils souhaitent financer et ceux qu'ils ne souhaitent pas financer.

\section{DISCUSSION}

Lors de l'évaluation des dossiers de candidature, une tension fondamentale apparaît entre les deux missions des agents des services partenariat qui sont la création de partenariats et l'application des règles de collaboration. Pour sortir de cette tension, les agents débattent à propos des critères de sélection jusqu’à sceller un accord. Et, en ce sens, ils utilisent leur pouvoir discrétionnaire pour juger les dossiers en conséquence. Les évaluateurs échangent des arguments de différents types à la fois sur le contenu du dossier (le respect des critères de sélection, le type de projet et ses modalités, la qualité du dossier, le caractère 
nouveau et innovant, et le prix en dernier lieu) et sur l'étiquette réputationnelle de l'opérateur (les collaborations antérieures, les résultats obtenus à propos des chômeurs, le professionnalisme, la fiabilité, son tempérament, etc.).

Les évaluateurs font intervenir la connaissance interpersonnelle qu'ils ont des opérateurs. Au-delà du degré de compétition et de sélectivité des dossiers, les évaluateurs du Forem font plus souvent intervenir des arguments révélant la proximité avec les opérateurs (connaissance interpersonnelle et familiarité) que leurs homologues chez Actiris. Cela peut s'expliquer du fait qu'au sein du Forem, la gestion du partenariat ${ }^{6}$ s'effectue de manière individuelle, ce qui n'est pas le cas chez Actiris où elle prend une dimension collective. L'encadrement individuel des opérateurs pour la mise en place de leur prestation offre aux agents une certaine proximité avec ceux-ci donc ils en ont une très bonne connaissance interpersonnelle.

Selon les cas, les agents avancent des arguments en faveur ou en défaveur du dossier de candidature. Certains dossiers seraient a priori à exclure puis, à force d'échange d'arguments, les agents trouvent un compromis ou un arrangement donc ils les jugent positivement. Le cas inverse peut également se produire. Parfois, les évaluateurs peuvent échouer dans la recherche d'un arrangement car la règle ne peut être modulée à souhait dans le sens où l'esprit de la règle doit être respecté puis, les opérateurs doivent être traités avec équité.

Les agents doivent «réinterpréter » les critères du cahier des charges de l'appel à projets afin de juger positivement un certain nombre de dossiers pour établir des collaborations avec les opérateurs privés. Ils effectuent des «accommodements des règles » (Dodier, 1989) pour être en mesure de répondre à leur mission de service public. C'est en réinterprétant les critères de sélection que les évaluateurs sont en mesure de pouvoir réaliser, selon les cas, des compromis ou faire des arrangements. Les différents cas montrent qu'«une règle n’est pas une solution toute faite mais une heuristique au service d'un processus d’apprentissage collectif » (Favereau, 1994, p. I32).

6 Pour d'amples informations sur l'impact des modèles de partenariat sur la collaboration, voir Remy, 2015. 


\section{CONCLUSION}

Cet article est centré sur la sélection des opérateurs privés par les services publics de l'emploi au moment où ils doivent décider de déléguer leur mission d'accompagnement et de formation des demandeurs d'emploi à des opérateurs privés. Cette délégation s'effectue dans le cadre d’appels à projets lancés par les SPE en Belgique francophone (Bruxelles et la Wallonie). Pour ce faire, les agents des services partenariats des SPE ont notamment pour tâche d'examiner les dossiers de candidature déposés par les opérateurs. Ils doivent trouver un accord pour chaque dossier et lui attribuer un jugement. Les dossiers sont parfois incomplets, incohérents, erronés mais aussi de mauvaise qualité. Ils ne répondent pas à tous les critères de sélection du cahier des charges de l'appel à projets. Les agents qui portent leur casquette d'évaluateur à ce moment-là peuvent aussi avoir des avis opposés à propos du jugement à octroyer à un dossier. Ils débattent alors à coup d'argument pour arriver à sceller un accord.

L’analyse montre que les agents utilisent leur pouvoir discrétionnaire pour faire des compromis et trouver des arrangements pour certains dossiers. Les critères de sélection ne sont pas appliqués tels quels mais ils font l'objet d'une interprétation par les agents. Certains dossiers qui devaient être exclus au départ sont, à la suite du débat, jugés positivement; et inversement. Les arguments mobilisés concernent tant le dossier de candidature (contenu, qualité, caractère innovant, etc.) que l'étiquette réputationnelle de l'opérateur qui s’appuie sur la qualité des prestations antérieures (résultats obtenus à propos des chômeurs, professionnalisme, fiabilité, relation avec les agents du SPE, tempérament, etc.). Quand les agents n'arrivent pas à octroyer un jugement au dossier et qu'ils manquent d'argument en faveur et en défaveur du dossier, ils se demandent s'ils souhaitent ou non établir une collaboration avec l'opérateur en question et soutenir financièrement ce projet. Et, c'est à partir de l'affirmation ou de la négation que les agents construisent le jugement et l'argumentation qui va de pair. On peut voir ainsi que les règles liées à la sélection des opérateurs privés par les agents des SPE s'inscrivent dans un processus d’apprentissage collectif. 


\section{BIBLIOGRAPHIE}

BATIFOULIER Ph., 200I, Théorie des conventions, Paris, Economica.

BOLTANSKI L., 1990, L'Amour et la Justice comme compétences, Paris, Métailié.

- et THÉVENOT L., 1991, De la justification, Paris, Gallimard.

CHASERANT C. et THÉVENON O., 200I, «Aux origines de la théorie économique des conventions: prix, règles et représentations », in BATIFOULIER, 200I, p. 35-61.

CORTÉSÉRO R., KERBOURC'H S., MÉLO D. et POLI A., 2013, «Recruteurs sous tensions. Discrimination et diversité au prisme de registres argumentaires enchevêtrés», Sociologie du travail, 55, p. 431-453.

DIVAY S., 2009, «Nouveaux opérateurs privés du service public de l'emploi.

Les pratiques des conseillers sont-elles novatrices?», Travail et Emploi, I19, p. 37-49.

DUBOIS V., 20I3, «Introduction. The Sociology of Administrative Work, a Study of "Street-Level Bureaucracy" à la Française», in J.-M. Eymeri-Douzans et G. Bouckaert (dir.), La France et ses administrations. Un état des savoirs, Bruxelles, Bruylant-De Boeck, p. 169-176.

DODIER N., 1989, «Le travail d'accommodation des inspecteurs du travail en matière de sécurité », in L. Boltanski et L. Thévenot (dir.), Justesse et justice dans le travail, Paris, PUF, p. 281-306.

FAVEREAU O., 1995. «Apprentissage collectif et coordination par les règles: application à la théorie des salaires », in N. Lazaric et J.-M. Monier (dir.), Coordination économique et apprentissage des firmes, Paris, Economica, p. 23-38.

GEORGES N., 2007, «L'externalisation de l'accompagnement des demandeurs d'emploi: modalités d'un marché en plein essor», Paris, CEE, Document de travail, 81.

LASCOUMES P., 1990, «Normes juridiques et mise en œuvre des politiques publiques», L'Année sociologique, 40, p.40-71.

LIPSKY M., 1980, Street-level Bureaucracy: The Dilemmas of Individuals in Public Service, New York, Russel Sage Foundation.

NACHI M., 200I, «La vertu du compromis: dimensions éthique et pragmatique de l'accord», Revue interdisciplinaire d'études juridiques, 46, p. 81-1।0. 
-, 2007, «Arrangement au présent, compromis au futur. Les «cadres de l'expérience» d'un groupe de jeunes garçons dans le contexte tunisien», in M. Briviglierie et V. Cicchelli (dir.), Adolescences méditerranéennes. L'espace public à petits pas, Paris, L'Harmattan, p. 315-338.

RÉBÉRIOUX A., BIENCOURT O. et GABRIEL P., 200I, «La dynamique des conventions entre consensus et conflit», in BATIFOULIER, 200I, p. 253-277

REMY C., 2013, « Les chargés des relations partenariales et ses clients: Significations et usages de la confiance », Revue européenne de droit social, 20-3, p.67-98.

-, 2015, « La création de partenariats par les services publics de l'emploi belges et suisses: Quels sont les impacts du modèle de gestion du partenariat sur la collaboration?», Politiques et management public, 32-I, p. 63-77.

ROUSSEAU Y., 2005, «Du monopole public de placement à un nouveau service public de l'emploi», Droit social, 4, p.456-465.

VIVÈS C., 2013, «L'institutionnalisation du recours aux opérateurs privés de placement au cœur des conflits de régulation du service public de l'emploi», Thèse de l'université de Nanterre.

\section{ANNEXE}

Figure I. Schéma de l'appel à projet pour le Forem

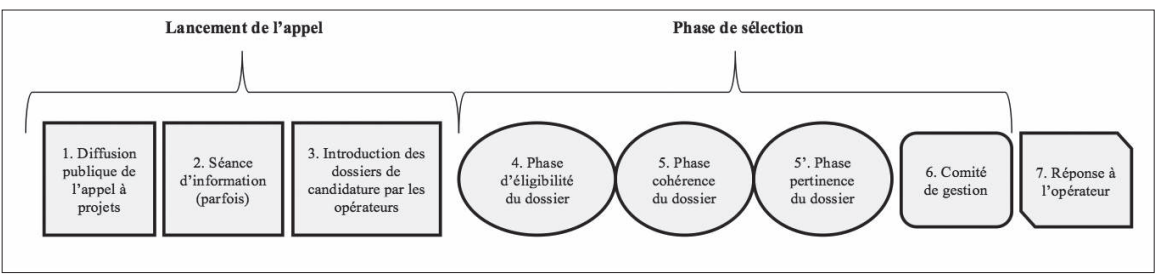

Figure 2. Schéma de l'appel à projet pour Actiris

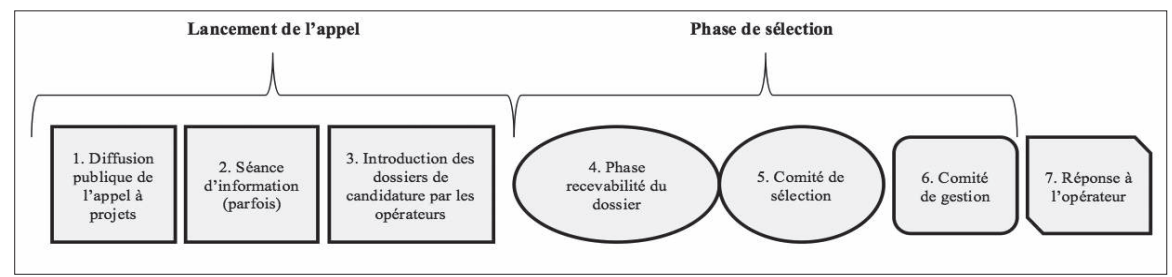

